\title{
High field - low energy muon ionization cooling channel
}

\author{
Hisham Kamal Sayed and Robert B. Palmer \\ Brookhaven National Laboratory, Upton, New York 11973, USA \\ David Neuffer \\ Fermi National Laboratory, Batavia, Illinois 60510, USA \\ (Received 27 April 2015; published 4 September 2015)
}

\begin{abstract}
Muon beams are generated with large transverse and longitudinal emittances. In order to achieve the low emittances required by a muon collider, within the short lifetime of the muons, ionization cooling is required. Cooling schemes have been developed to reduce the muon beam $6 \mathrm{D}$ emittances to $\approx 300 \mu \mathrm{m}-\mathrm{rad}$ in transverse and $\approx 1-1.5 \mathrm{~mm}$ in longitudinal dimensions. The transverse emittance has to be further reduced to $\approx 50-25 \mu \mathrm{m}$-rad with an upper limit on the longitudinal emittance of $\approx 76 \mathrm{~mm}$ in order to meet the high-energy muon collider luminosity requirements. Earlier studies of the transverse cooling of low energy muon beams in high field magnets showed a promising performance, but did not include transverse or longitudinal matching between the stages. In this study we present the first complete design of the high field-low energy ionization cooling channel with transverse and longitudinal matching. The channel design was based on strong focusing solenoids with fields of 25-30 T and low momentum muon beam starting at $135 \mathrm{MeV} / \mathrm{c}$ and gradually decreasing. The cooling channel design presented here is the first to reach $\approx 50$ micron scale emittance beam. We present the channel's optimized design parameters including the focusing solenoid fields, absorber parameters and the transverse and longitudinal matching.
\end{abstract}

DOI: 10.1103/PhysRevSTAB.18.091001

PACS numbers: 41.75.Ak, 41.85.Ja, 41.85.Ct

\section{INTRODUCTION}

The muon collider offers significant advantages over an electron machine as a lepton collider. Muons, with a mass 200 times heavier then electrons, have an increased crosssection for Higgs production, by $\approx$ four orders of magnitudes [1]. More importantly, a muon collider does not suffer from some of the difficulties associated with high energy electrons, e.g., synchrotron radiation and beamstrahlung. This allows us to use multiturn accelerators with much lower cost and size.

However, producing and accelerating high intensity muon beams is a challenging task. Muon beams are produced from pion beam decay. Pions are generated by the collision of multi-GeV proton beams with a target. The resulting pion beam has large transverse and longitudinal emittances, which require reduction in all six phase-space dimensions to fit within the longitudinal and transverse acceptances of the muon accelerator. In addition to these challenges, muons have a very small lifetime of $2.2 \mu \mathrm{s}$, which requires fast and aggressive cooling and acceleration.

Muon colliders were first proposed by Budker in 1969, and later more detailed design concepts were proposed in [2-4]. Muon accelerators must deliver high luminosity

Published by the American Physical Society under the terms of the Creative Commons Attribution 3.0 License. Further distribution of this work must maintain attribution to the author(s) and the published article's title, journal citation, and DOI. muon beams with transverse emittances less than $50 \mu \mathrm{m}-\mathrm{rad}$ for high-energy muon collider rings. Reduction of the initial large 6D transverse emittance of muon beam is achievable only by ionization cooling, where muon momenta are reduced in all directions as they pass through the absorber material. Absorbers are followed by sets of rf cavities which restore only the longitudinal component of the momentum. This process cools only the transverse emittance directly, but when combined with emittance exchange it also reduces longitudinal emittance, providing $6 \mathrm{D}$ phase space cooling of the muon beam.

A complete design concept of a high intensity muon collider from production to collision was given in [5]. The design has five major components; the high power target for pion production [6], the rf bunching, the $6 \mathrm{D}$ cooling, the fast acceleration, and finally the injection into the collider ring. The muon collider design starts with the muon front end, consisting of the pion production target and the bunching channel [6-9] that yields bunch trains of both muon signs.

The muon front end is followed by the 6D cooling lattices which reduce the longitudinal emittance until it becomes possible to merge the trains into single bunches, one of each sign. Further cooling in all dimensions is applied to the single bunches in the following 6D cooling lattices.

A complete scheme of muon cooling for a muon collider was presented in [5]. In this scheme muons of both signs are cooled transversely in a linear channel using $\mathrm{LiH}$ absorbers in a periodic $2.8 \mathrm{~T}$ solenoid lattice with $\mathrm{rf}$ cavities for acceleration. This is followed by a 6-D cooling 
channel which uses wedge shaped liquid hydrogen absorbers, tilted solenoids for focusing and dispersion generation, and rf cavities for acceleration. Since the collider luminosity is proportional to $N^{2}$, where $N$ is the number of muons per bunch, it is desirable to have relatively few bunches with larger $N$. A bunch merging channel would therefore follow the initial 6D cooling [5].

A second 6D cooling channel follows the bunch merging channel. The second 6D cooling channel will have much stronger solenoid focusing and should reduce the muon beam emittance to $\epsilon_{T}=280 \mu \mathrm{m}-\mathrm{rad}$ and $\epsilon_{L}=1.5 \mathrm{~mm}$.

Several 6D ionization cooling channel designs have been proposed [10-13]. Simultaneous transverse and longitudinal cooling is achieved by using wedge-shaped absorber materials or a differential path length in an absorber region with nonvanishing dispersion.

In $6 \mathrm{D}$ cooling channels particles with different momenta will experience differing path lengths inside the absorber due to the dispersion, leading to reduction in the longitudinal emittance. These 6D ionization cooling channels operate with muon beams of central momentum $\approx 220 \mathrm{MeV} / \mathrm{c}$ and cannot provide cooling beyond $\epsilon_{T} \approx 280 \mu \mathrm{m}$-rad and $\epsilon_{L} \approx 1.5 \mathrm{~mm}[11-13]$.

The final stage of the muon collider ionization cooling is a transverse only cooling channel [14]. It utilizes liquid hydrogen absorbers inside strong focusing solenoids. The reduction in the transverse emittance takes place at the expense of heating the longitudinal emittance.

The final transverse cooling channel for the muon collider was proposed in $[5,14,15]$. The design proposed cooling low-energy muons with liquid $\mathrm{H}_{2}$ absorbers in 30-50 T solenoids. The reported design concept showed that a final emittance of $25 \mu \mathrm{m}$ is achievable, assuming ideal matching between the stages and lowering the beam energy down to $5 \mathrm{MeV}$.

The focus of this work is to present a complete design of the high field-low energy transverse cooling channel for use in the final cooling segment of the muon collider. The design reported here is based on a 25-30 T focusing solenoids. The design includes complete and realistic transverse and longitudinal matching between the stages. The maximum field strength in the channel was limited to $30 \mathrm{~T}$ to reduce the cost and adhere to the current technological limitations of high field magnets.

The channel works in a momentum regime that starts at $135 \mathrm{MeV} / \mathrm{c}$ and decreases gradually. At such low momenta, the longitudinal emittance rises from the heating effect of the negative slope of energy loss versus energy. Starting with initial emittances achieved by $6 \mathrm{D}$ cooling channels, we show that the channel design can achieve transverse emittances of $55 \mu \mathrm{m}$-rad within a longitudinal acceptance of $76 \mathrm{~mm}$-rad. This allows the use of this channel design in muon collider final cooling stages.

In the following we first discuss the physics and motivation of ionization cooling at low energy (Sec. II).
We then review and the discuss feasibility and parameters of high field magnets (Sec. III). Following that we give the details of our final cooling channel design (Sec. IV), followed by simulation and tracking studies (Sec. V), establishing the cooling channel performance.

\section{IONIZATION COOLING AT LOW ENERGIES}

Inside the absorber material, the reduction in beam momenta providing transverse cooling is opposed by the multiple Coulomb scattering. Multiple Coulomb scattering in the absorber material limits the ionization cooling and acts as a heating element. Strong focusing fields at the absorber location help in minimizing the longitudinal heating effect. Equation (1) gives the rate of change of the normalized transverse emittance inside an absorber material [16]

$$
\frac{d \epsilon_{N}}{d s}=-\frac{\epsilon_{N}}{\beta^{2} E} \frac{d E}{d s}+\frac{\beta_{\perp} E_{s}^{2}}{2 \beta^{3} m c^{2} L_{R} E}
$$

where $\beta_{\perp}(\approx 2 P / 0.3 B)$ is the transverse betatron function of the beam, $\beta=v / c, L_{R}$ is the radiation length of the absorber material, $E=\gamma m c^{2}$ is the total particle energy, and $E_{s}=13.6 \mathrm{MeV}$.

The energy loss in material $d E / d s$ is given by the Bethe-Bloch formula [17]

$$
\frac{d E}{d s}=4 \pi N_{A} \rho r_{e}^{2} m_{e} c^{2} \frac{Z}{A}\left[\frac{1}{\beta^{2}} \ln \left(\frac{2 m_{e} c^{2} \gamma^{2} \beta^{2}}{I(Z)}\right)-1-\frac{\delta}{2 \beta^{2}}\right],
$$

where $N_{A}$ is Avogadro's number, $A, Z$ are the atomic weight and number of the absorber material of density $\rho$. and $m_{e}$ and $r_{e}$ are the electron mass and classical radius, respectively. The density effect factor $\delta \approx 0$. The material ionization energy $I(Z)$ in eV can be estimated by [16]

$$
I(Z)=16 Z^{0.9} .
$$

When both the ionization cooling and the multiple scattering heating in the absorber material come to equilibrium, the normalized transverse emittance of the beam cannot be reduced beyond the value of the equilibrium emittance given by

$$
\epsilon_{\mathrm{equ}, N}=\frac{\beta_{\perp} E_{s}^{2}}{2 \beta m c^{2} L_{R}(d E / d s)},
$$

which gives the minimum achievable emittance in any absorber material in a simpler form:

$$
\epsilon_{\min , N} \propto \frac{E}{B L_{R}(d E / d s)} .
$$

For a given absorber material $\epsilon_{\min , N}$ depends on the beam energy $E$ and the focusing strength $B$. Reducing the beam 
energy gradually in the cooling channel with strong focusing fields can provide a reduction in the transverse emittance to values in the range of 60-25 $\mu \mathrm{m}$-rad. Muon ionization cooling to emittances below $50 \mu \mathrm{m}$-rad can be achieved with liquid hydrogen absorbers placed in high field solenoids which have small bores.

The disadvantage of the ionization cooling of low energy muons is the increase in the longitudinal emittance. At low energies the increase of the energy loss with reduced energy heats the beam longitudinally. The change of the rms energy spread of the beam during ionization cooling is given by

$$
\frac{d \sigma_{E}^{2}}{d s}=-2 \frac{\partial(d E / d s)}{\partial E} \sigma_{E}^{2}+\frac{d\left\langle\Delta E_{\mathrm{RMS}}^{2}\right\rangle}{d s} .
$$

The second term of the last equation represents the random fluctuations in the particle energy loss, which is a heating effect. In the long path length Gaussian-distribution limit, this term is given in $(\mathrm{MeV} \mathrm{cm})^{2} / \mathrm{g}$ by [16]

$$
\frac{d\left\langle\Delta E_{\mathrm{RMS}}^{2}\right\rangle}{d s} \approx 0.157 \rho \frac{Z}{A} \gamma^{2}\left(1-\frac{\beta^{2}}{2}\right) .
$$

As the muon beam transverse emittance is reduced, the longitudinal emittance increases accordingly. We aim to limit the longitudinal heating to within the longitudinal acceptance of the muon acceleration chain. Acceleration to high energy reduces the relative momentum spread $\delta P / P$ and improves the longitudinal acceptance.

Figure 1 shows an analytical estimation of the minimum equilibrium emittance achieved in the liquid hydrogen absorber using focusing solenoid fields of 20, 30, $40 \mathrm{~T}$. By reducing the muon beam kinetic energy and using

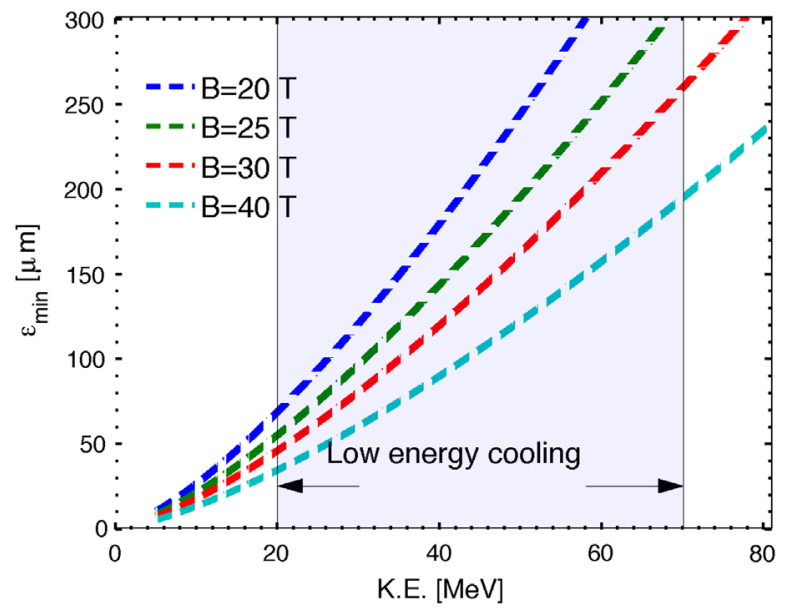

FIG. 1. Analytical estimate of the minimum equilibrium emittance achieved in liquid hydrogen absorber using focusing solenoid fields of 20,30, $40 \mathrm{~T}$. Reducing the muon beam kinetic energy and using strong focusing field can achieve very small emittance values down to $25 \mu \mathrm{m}$-rad.
TABLE I. $50 \mathrm{~T}$ high-field magnet parameters.

\begin{tabular}{lccc}
\hline \hline $\begin{array}{l}\text { Magnet } \\
\text { length [m] }\end{array}$ & $\begin{array}{c}\text { Inner } \\
\text { radius [m] }\end{array}$ & $\begin{array}{c}\text { Coil } \\
\text { thickness [m] }\end{array}$ & $\begin{array}{c}\text { Current density } \\
\left.\text { I/A [A/mm }{ }^{2}\right]\end{array}$ \\
\hline 0.317 & 0.025 & 0.029 & 164.26 \\
0.337 & 0.055 & 0.041 & 142.43 \\
0.375 & 0.098 & 0.056 & 125.88 \\
0.433 & 0.157 & 0.067 & 119.07 \\
0.503 & 0.228 & 0.120 & 85.99 \\
0.869 & 0.355 & 0.089 & 39.60 \\
0.868 & 0.454 & 0.104 & 44.30 \\
0.992 & 0.575 & 0.252 & 38.60 \\
\hline \hline
\end{tabular}

strong focusing fields one can achieve very small emittance values, down to $\approx 25 \mu \mathrm{m}$-rad.

\section{HIGH FIELD MAGNETS}

In this section we will briefly describe two possible highfield magnets that can be used in the cooling channel. A high field magnet was proposed in [18], it has a set of eight coaxial superconducting solenoid coils. It was designed to deliver peak fields up to $50 \mathrm{~T}$. The coils parameters and currents are given in Table I and the schematic of the magnet is shown in Fig. 2. In order to provide peak fields ranging from 30 to $25 \mathrm{~T}$, the current densities are scaled accordingly. These coils were used in the simulation of the cooling channel.

Another variety of high field magnet design, which is an all superconducting $45 \mathrm{~T}$ solenoid, was presented in [19]. The conceptual design of the $45 \mathrm{~T}$ solenoid is based on Bi-2223 HTS tape, and the magnet operates at $4.2 \mathrm{~K}$ to take advantage of the high current carrying capacity at that temperature. An outer $\mathrm{Nb} 3 \mathrm{Sn}$ shell surrounds the HTS solenoid.

Another $45 \mathrm{~T}$ hybrid magnet composed of a $33.5 \mathrm{~T}$ resistive magnet nested in an $11.5 \mathrm{~T}$ outsert is currently

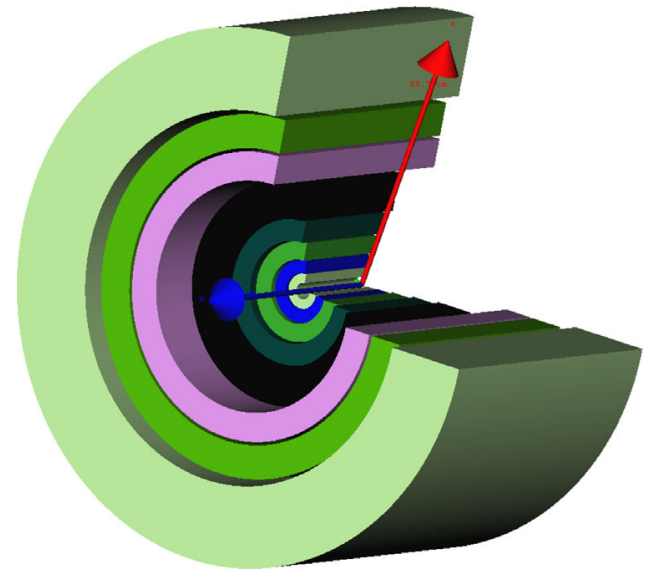

FIG. 2. A set of eight superconducting coaxial coils providing a peak field of $50 \mathrm{~T}$. The inner radius of the smallest coils is $0.025 \mathrm{~m}$. 


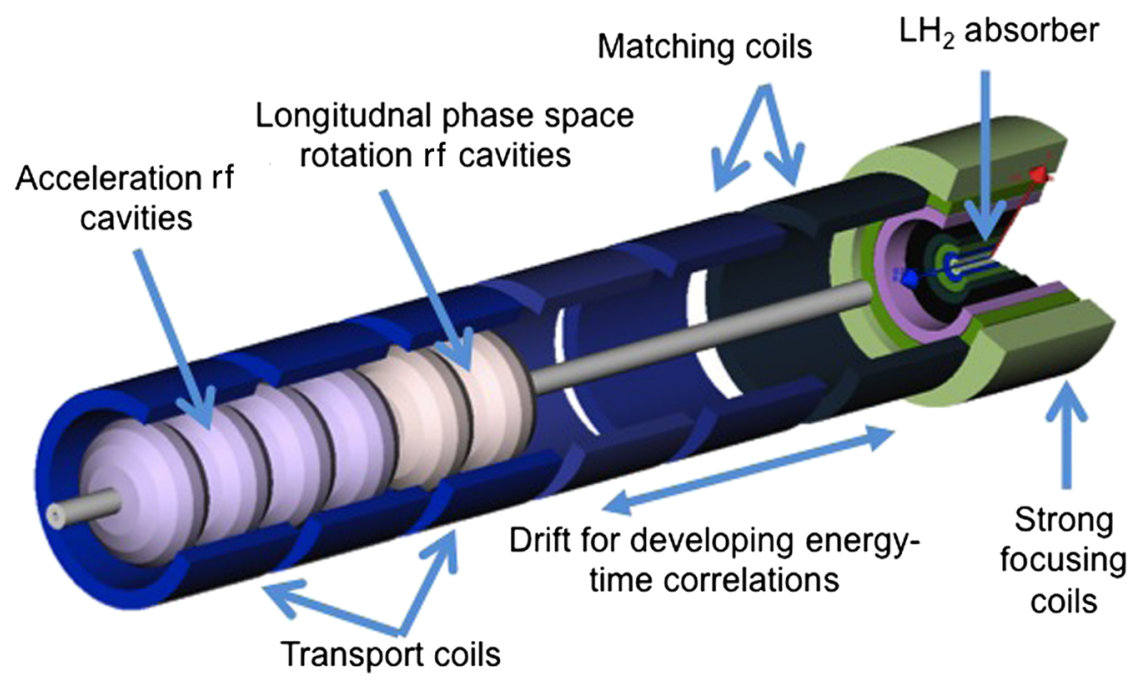

FIG. 3. Schematic of the elements of one ionization cooling stage. Each stage starts with strong coaxial focusing coils which enclose the $\mathrm{LH}_{2}$ absorber, followed by matching coils, energy-phase rotation rf cavities, and acceleration rf cavities.

operating at the National High Field Magnet Laboratory "NHFML" [20]. The disadvantage of this magnet is its large power consumption, and such large power consumption might not suit our needs. We would like to note that a $27 \mathrm{~T}$ solenoid using an $8 \mathrm{~T}$ YBCO insert at $4 \mathrm{~K}$ has recently been successfully tested at the same facility.

The magnet design details do not affect the cooling channel performance. We have used a scaled-down version of the $50 \mathrm{~T}$ magnet design in our simulations. A more simple superconducting magnet design with 25-30 T peak field will deliver the same ionization cooling performance.

\section{CHANNEL DESIGN}

The high-field low-energy cooling channel is composed of 16 stages in a straight section. Each stage consists of five major components: A set of coaxial strong focusing solenoid coils, liquid hydrogen absorber, two matching sections, longitudinal phase space rotation rf cavities, and acceleration rf cavities. Figure 3 shows a schematic of a single stage. A schematic of all of the 16 stages is shown in Fig. 4.

The muon beam momentum is reduced gradually along the channel to reduce the value of the equilibrium emittance and achieve the desired cooling. As a result each cooling stage has its own set of unique design parameters optimized for the momentum band at which the stage operates.

\section{A. Individual stage design}

Each stage contains a cylindrically shaped liquid hydrogen absorber placed inside the innermost coil of the high field magnet where $\beta_{\perp}$ has a minimum value. Figure 5 shows the $\beta_{\perp}$ inside the absorber for each stage. Such absorbers are feasible [21]. The strong focusing solenoid coils are preceded and followed by a set of matching coils to match the beam transport from a $3.5 \mathrm{~T}$ constant focusing field to the peak 30-25 T value while mitigating chromatic effects.

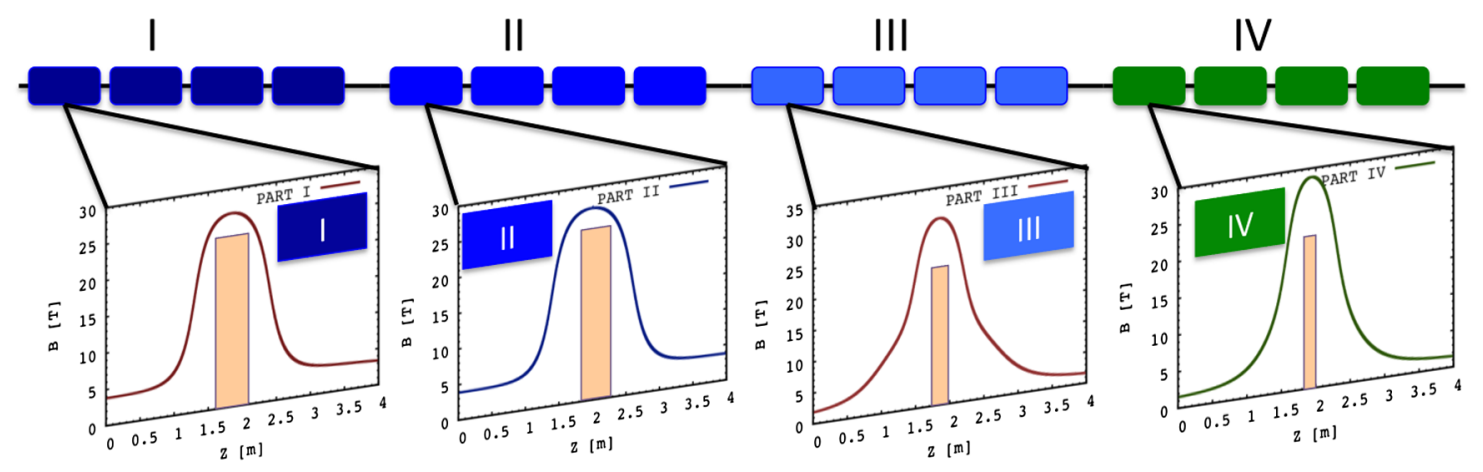

FIG. 4. A layout schematic of 16 stages of the cooling channel. The colored boxes in the top represent the cooling stages (a detailed schematic of one stage is shown in Fig. 3.). The bottom figures show a sample of the on-axis field of the strong focusing solenoid; the shaded areas show the corresponding absorbers lengths. 


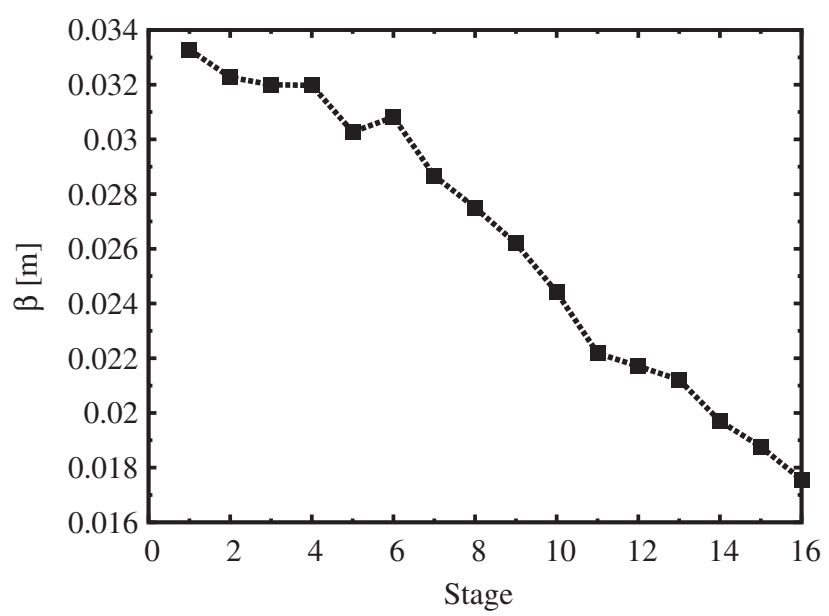

FIG. 5. Transverse Twiss $\beta$-function at the center of the strong focusing solenoid in each stage of the 16 stages.

Due to the fact that the beam mean momentum before passing through the absorber is different from that after exiting the absorber, the matching coils are asymmetric. The matching is done by propagating a set of $\beta_{\perp}$-functions for different momenta through the absorber material to account for the energy loss and increased energy spread. The muon momenta were chosen to represent the momentum band of the muon beam at each stage. An automated optimization algorithm was used to match the $\beta_{\perp}$-functions in and out of the absorber [22].

The matching segment has no rf cavities and was utilized to develop an energy-time correlation required for the energy-phase rotation. The length of the drift space between the absorber and the first rf cavity controls the amount of energy-phase rotation and hence the bunch length and energy spread after rotation. The drift length was optimized for each individual stage to reduce the energy spread and achieve the required cooling in the following stage. The first set of rf cavities after the drift is set to have zero phase to rotate the longitudinal phase space of the muon beam. Another set of rf cavities following the phase rotation section is used to accelerate the muon beam to the required energy for the following cooling stage. The accelerating rf cavities frequencies and phases are optimized to match the bunch length at every stage.

Each cooling stage performance was optimized using an automatic optimization algorithm based on tracking of a large statistical ensemble of particles. The optimization included the transverse and longitudinal matching between the stages. The optimization objectives were set to maximize cooling and minimize particle losses.

A set of field flip matches are inserted between the stages to limit the accumulation of the canonical angular momentum [15]. The matching was performed by minimizing the transverse $\beta$-beat for particles within the required momentum band. The field flip locations are given in Table II.

\section{B. Design variation between the stages}

The initial energy, energy spread, and absorber length for every stage are optimized to minimize $\frac{-d \epsilon_{\|}}{d \epsilon_{\perp}}$. The beam energy is gradually reduced and the bunch length increases gradually along the channel. As a result each stage has its own set of matching coils, focusing coils, rf cavities frequencies, which are different from the other stages.

The selection of the on-axis field at the absorbers locations and the match in and out of the strong focusing portion has a major impact on the cooling performance of the channel. We adopted four differing focusing solenoid sets which were used throughout the channel according

TABLE II. Parameters of the high-field low-energy cooling channel.

\begin{tabular}{lccccccc}
\hline \hline $\begin{array}{l}\text { Stage } \\
{[\mathrm{N}]}\end{array}$ & $\begin{array}{c}\mathrm{P} \\
{[\mathrm{MeV} / \mathrm{c}]}\end{array}$ & $\begin{array}{c}\text { Energy spread } \\
\sigma_{E}[\mathrm{MeV}]\end{array}$ & $\begin{array}{c}\mathrm{LH}_{2} \text { thickness } \\
{[\mathrm{cm}]}\end{array}$ & $\begin{array}{c}\text { Drift length } \\
{[\mathrm{m}]}\end{array}$ & $\begin{array}{c}\text { rf length } \\
{[\mathrm{m}]}\end{array}$ & $\begin{array}{c}\text { rf frequency } \\
{[\mathrm{MHz}]}\end{array}$ & $\begin{array}{c}\text { Field flip } \\
1\end{array}$ \\
\hline 135.0 & 2.29 & 65 & 0.434 & 2.25 & 325 & Yes \\
2 & 130.0 & 2.48 & 60 & 0.459 & 2.25 & 250 & Yes \\
3 & 129.0 & 2.78 & 60 & 0.450 & 2.5 & 220 & No \\
4 & 129.0 & 3.10 & 59 & 0.458 & 2.5 & 201 & No \\
5 & 122.0 & 3.60 & 57 & 1.629 & 5.0 & 201 & Yes \\
6 & 124.0 & 4.90 & 53 & 2.22 & 4.5 & 180 & No \\
7 & 116.0 & 3.40 & 42 & 2.21 & 3.25 & 150 & No \\
8 & 111.0 & 3.90 & 40 & 2.0 & 3.5 & 150 & No \\
9 & 106.0 & 3.50 & 40 & 3.13 & 5.0 & 125 & Yes \\
10 & 98.0 & 3.07 & 35 & 3.13 & 5.0 & 120 & No \\
11 & 89.4 & 3.11 & 20 & 3.12 & 5.0 & 110 & No \\
12 & 87.9 & 2.76 & 20 & 3.1 & 8.0 & 100 & No \\
13 & 85.9 & 2.67 & 20 & 3.0 & 7.5 & 100 & Yes \\
14 & 79.7 & 3.08 & 15 & 2.7 & 7.0 & 70 & No \\
15 & 71.1 & 4.0 & 15 & 2.6 & 6.0 & 50 & No \\
16 & 71.0 & 3.80 & 13 & 2.5 & 6.0 & 20 & No \\
17 & 70.0 & 3.80 & 10 & $\ldots$ & $\ldots$ & 20 & $\ldots$ \\
\hline \hline
\end{tabular}


to the value of the transverse beam emittance and energy spread. The strength of the focusing field and the matching coils are optimized for each stage individually.

At the initial stages where the transverse emittance is large $\sim 300 \mu \mathrm{m}$-rad we would like to avoid the longitudinal emittance growth from the amplitude dependent transit time effect [6]. The field integral is minimized to provide the required focusing for reducing the $\epsilon_{\text {equ }}$ and match the length of the absorber. The peak magnetic field is designed to have a short decline to the transport field value of $3.5 \mathrm{~T}$. In the later stages the energy of the beam is further decreased and hence the growth of the energy spread per unit absorber length is larger. Shorter absorbers are used and the cooling will require a stronger field of $30 \mathrm{~T}$.

In the first four stages (first quarter of the channel) $\mathrm{LH}_{2}$ absorbers are long which requires a longer section at peak field. It is desired to limit the field integral to avoid unnecessary increase in the bunch length due to transverselongitudinal coupling in the high field solenoids [6]. The on-axis field profile for each stage was optimized to meet these two requirements. See Fig. 6(a).

In the second quarter (stages 5-8), $\mathrm{LH}_{2}$ absorbers have a moderate length and the beam has relatively smaller transverse amplitudes and already long bunch length. The on-axis field in this case will have a slightly more adiabatic match to the lower field and a slightly smaller peak field length. See Fig. 6(b).

In the third and fourth quarters (stages 9-16), the $\mathrm{LH}_{2}$ absorbers have a medium thickness. The beam has slightly larger energy spread which will lead to unwanted chromatic effects. A more adiabatic match to the peak field was required to compensate for the chromatic effects and has a small impact on the already long bunch length. See Figs. 6(c) and 6(d).

As the bunch goes through the energy phase rotation section in each stage it gets longer. We start the simulation of the channel with $\sigma_{t}=5 \mathrm{~cm}$ and the final bunch length is $1.8 \mathrm{~m}$. The $\mathrm{rf}$ frequencies were chosen to keep the bunch length $\sigma_{c t}<\lambda / 20$. Table II shows if parameters used in every stage. The gradients assumed maximum surface fields $\approx \sqrt{f}$, and assuming reentrant vacuum cavities with surface to accelerating gradients $\propto f^{0.75}$. Only the last accelerating stage required an induction linac.

In the first stage a set of $325 \mathrm{MHz}$ rf cavities are used and are placed inside the $3.5 \mathrm{~T}$ transport coils. In the later stages the bunch gets longer, which mandates the use of lower rf frequencies. The size of the rf cavities increases, and the cell structure is switched to use smaller coils inside the rf field. The bunch length and the energy spread at different stages are shown in Fig. 7.

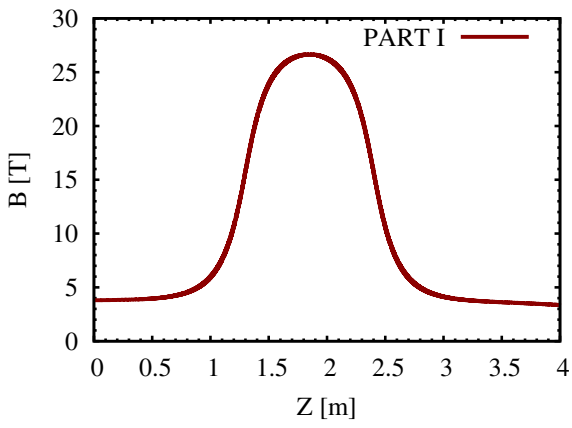

(a)

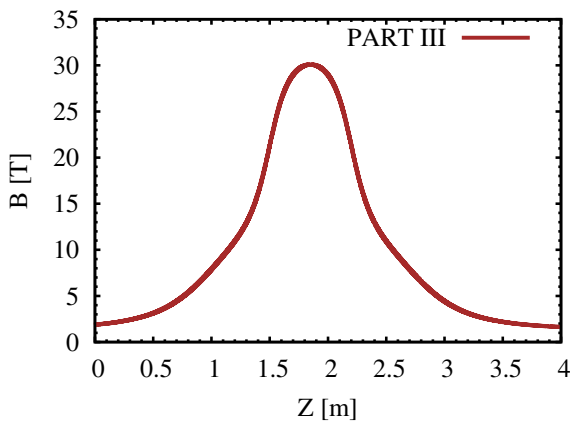

(c)

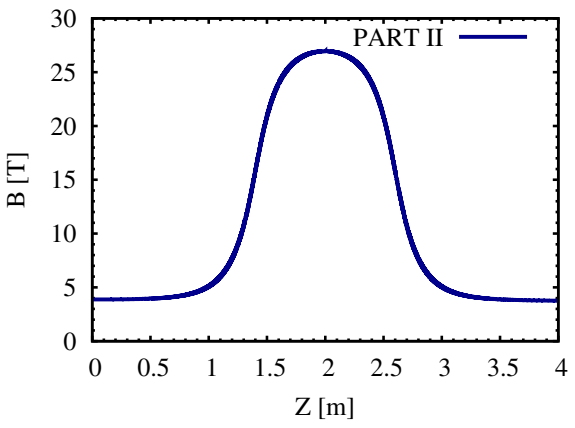

(b)

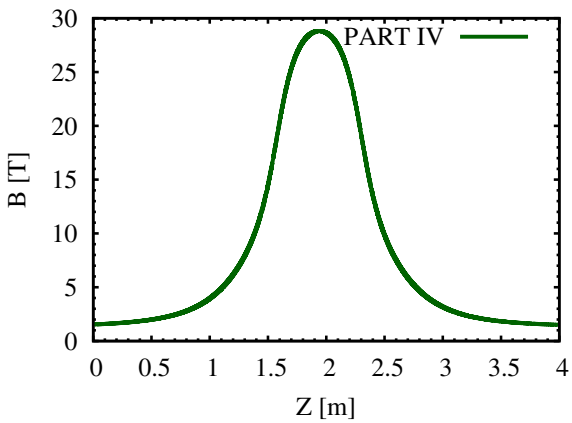

(d)

FIG. 6. On-axis field of four of the focusing solenoids used in the ionization cooling channel. (a) First quarter: Long absorbers. Limit the field integral to limit the transverse - longitudinal coupling and limit unnecessary increase in ${ }^{\sigma} t$, (b) Second quarter: Long absorbers but relatively smaller transverse amplitudes and already longer bunch length, (c) Third quarter: Medium absorber thickness. Larger energy spread will lead to unwanted chromatic effects and (d) Fourth quarter: Small absorber thickness. Very small transverse amplitudes. 


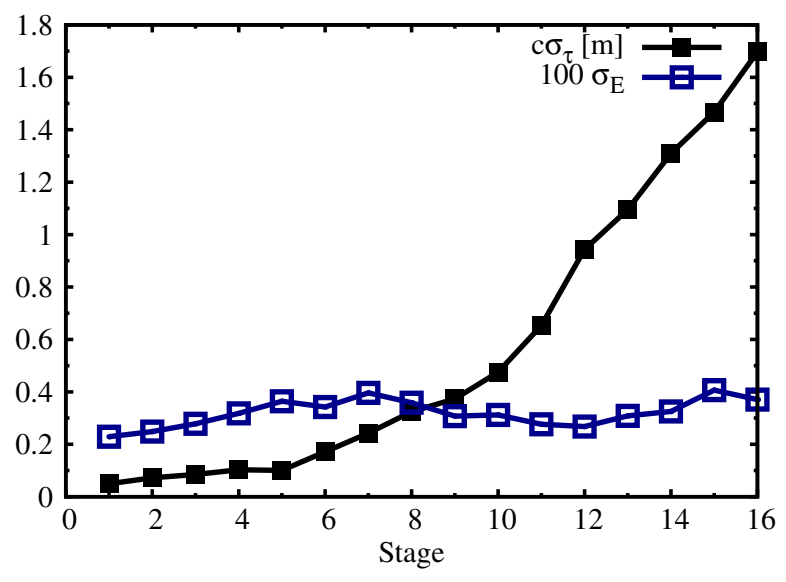

FIG. 7. Bunch length and energy spread in $\mathrm{MeV}$ across the channel.

\section{SIMULATION OF THE COOLING CHANNEL}

The high field-low energy cooling channel was simulated using the G4BEAMLINEA code [23], which is based on GEANT4 [24] physics libraries. The magnetic field of the solenoids was computed using a realistic configuration of coils and their current settings.

The rf cavities were modeled as cylindrical pillboxes running in the TM010 mode. A reference particle was used to determine each rf cavity's relative phase. The reference particle is a muon that is used as a clock to determine the entry times for each cavity in the channel. The reference particle is allowed to lose energy in absorber and gain energy during $\mathrm{rf}$ acceleration, so that it travels with the bunch.

G4BEAMLINE uses the GEANT4 physics model QGSP-BERT, and it includes all the relevant physical processes for simulating the low energy beam interaction with absorber material. This includes energy loss, straggling, multiple scattering, and muon decays.

In this simulations we used a Gaussian input beam with normalized transverse emittance $\epsilon_{\perp}$ of $300 \mu \mathrm{m}$-rad and longitudinal emittance $\epsilon_{L}$ of $1.5 \mathrm{~mm}$. The initial momentum distribution was generated with an average longitudinal momentum of $135.0 \mathrm{MeV} / \mathrm{c}$. Tracking was performed using an initial sample of $10^{7}$ particles matched and injected into the first ionization cooling stage.

\section{OPTIMIZATION OF THE CHANNEL PERFORMANCE}

Each stage performance was optimized automatically using multilayered parallel optimization algorithms [9,25]. The optimization was based on tracking of the muon beam through the whole cooling channel. The optimization was performed to improve both the transverse and the longitudinal motion as both are coupled during the cooling and matching. The optimization parameters included the focusing coils, matching coils, absorber lengths, drift lengths from the absorber to first energy-phase rotation cavity, and

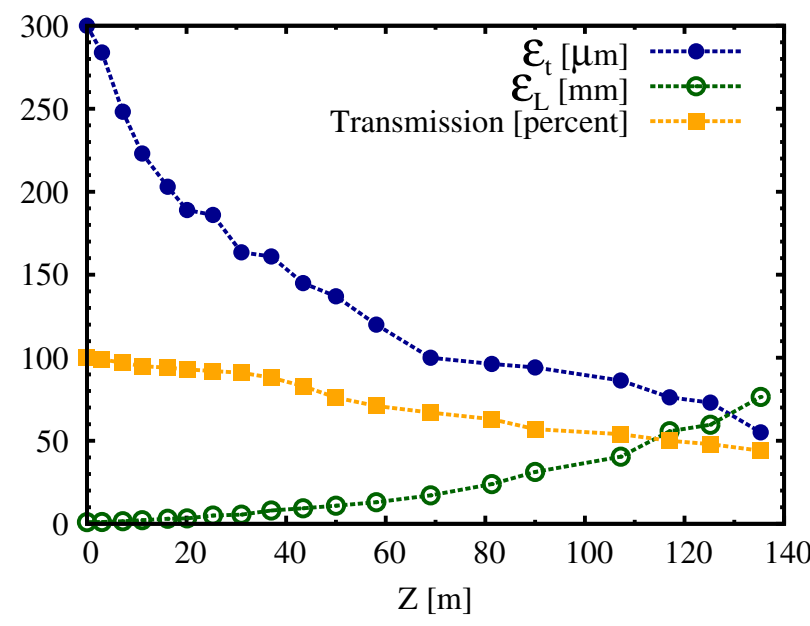

FIG. 8. Transverse and longitudinal emittance in the channel, along with the transmission which includes muon decay.

rf frequencies/phases. The optimized channel parameters are given in Table II. The transverse and longitudinal emittances evolution through the channel and the muon transmission are shown in Fig. 8. The channel was able to cool the muon beam from $300 \mu \mathrm{m}$-rad down to $55 \mu \mathrm{m}$-rad. The longitudinal emittance of the beam after cooling is $76 \mathrm{~mm}$.

\section{SUMMARY}

A high luminosity muon collider requires a very small transverse beam emittance. The muon beam, which is initially produced with a large transverse emittance, has to go through a series of 6D ionization cooling channels to reduce the emittance down to $300 \mu \mathrm{m}-\mathrm{rad}$. The $6 \mathrm{D}$ cooling channels are limited due to the compact nature of those channels and the limited equilibrium emittance values they can achieve while operating at momentum of $200 \mathrm{MeV} / \mathrm{c}$ with a maximum focusing strength of $\approx 15 \mathrm{~T}$. Further reduction in emittance requires reducing the operating energy and using much stronger fields, fields of the order of $25-30 \mathrm{~T}$.

In this work we presented the first complete channel with real matching of a high field-low energy cooling system. The maximum peak field considered in this study was limited to the current state of the art magnets, i.e., $30 \mathrm{~T}$ and the lowest beam momentum was $70 \mathrm{MeV} / \mathrm{c}$. The design parameters of the cooling channel were discussed in detail.

The channel shows promising performance as the final cooling stage for the muon collider. More work will be required to further reduce the transverse emittance to the $25 \mu \mathrm{m}$-rad desired for a very high-luminosity and highenergy muon collider.

It is worth mentioning that the channel performance is not dependent on the magnet design or the absorber outer geometry. Any magnet design that delivers the on axis fields discussed in Sec. IV will deliver the required cooling performance. 


\section{ACKNOWLEDGMENTS}

The authors are grateful to H. Kirk, S. Kahn, M. Palmer, R. Ryne, D. Stratakis, and R. Weggel for useful discussions. This research used resources of the National Energy Research Scientific Computing Center, which is supported by the Office of Science of the U.S. Department of Energy under Contract No. DE-AC02-05CH11231.

[1] D. M. Kaplan, Muon collider/neutrino factory: Status and prospects, Nucl. Instrum. Methods Phys. Res., Sect. A 453, 37 (2000).

[2] D. B. Cline, The scientific case for a Higgs boson $\mu^{+} \mu^{-}$ collider factory, AIP Conference Proceedings 441, 1 (1998).

[3] A. Skrinsky, in Proceedings of the 4th Int. Conf. on Physics Potential and Development at $\mu^{+} \mu^{-}$Colliders, San Francisco, CA, 1997 (F. Mills Publications, Salt Lake City, Utah, 1997), pp. 696-698.

[4] D. Neuffer and R. Palmer, in Proceedings of the Fourth European Particle Accelerator Conference EPAC 94, London, England (World Scientific, River Edge, NJ, 1994).

[5] R. B. Palmer, J. S. Berg, R. C. Fernow, J. C. Gallardo, H. G. Kirk, Y. Alexahin, D. N. Fermilab, S. A. Kahn, and D. J. Summers, in Proceedings of the 22nd Particle Accelerator Conference, PAC-2007, Albuquerque, NM (IEEE, New York, 2007).

[6] H. K. Sayed and J. S. Berg, Optimized capture section for a muon accelerator front end, Phys. Rev. ST Accel. Beams 17, 070102 (2014).

[7] C. T. Rogers, D. Stratakis, G. Prior, S. Gilardoni, D. Neuffer, P. Snopok, A. Alekou, and J. Pasternak, Muon front end for the neutrino factory, Phys. Rev. ST Accel. Beams 16, 040104 (2013).

[8] H. K. Sayed, J. S. Berg, H. Kirk, X. Ding, K. McDonald, and $\mathrm{C}$. Rogers, in Proceedings of the 4th International Particle Accelerator Conference, IPAC-2013, Shanghai, China, 2013 (JACoW, Shanghai, China, 2013).

[9] H. Sayed, J. Berg, H. Kirk, R. Palmer, D. Stratakis, K. McDonald, D. Neuffer, J. Qiang, and R. Ryne, in Proceedings of the North American Particle Accelerator Conference NAPAC2013, 2013, http://accelconf.web.cern .ch/AccelConf/pac2013/.

[10] R. Fernow and R. Palmer, Solenoidal ionization cooling lattices, Phys. Rev. ST Accel. Beams 10, 064001 (2007).

[11] R. Palmer, V. Balbekov, J. S. Berg, S. Bracker, L. Cremaldi, R. C. Fernow, J. C. Gallardo, R. Godang, G. Hanson,
A. Klier, and D. Summers, Ionization cooling ring for muons, Phys. Rev. ST Accel. Beams 8, 061003 (2005).

[12] K. Yonehara, Y. Derbenev, and R. Johnson, Helical channel design and technology for cooling of muon beams, AIP Conf. Proc. 1299, 658 (2010).

[13] D. Stratakis, R. C. Fernow, J. S. Berg, and R. B. Palmer, Tapered channel for six-dimensional muon cooling towards micron-scale emittances, Phys. Rev. ST Accel. Beams 16, 091001 (2013).

[14] R. B. Palmer, Muon collider progress, eConf. Proc. C090504, 652 (2009).

[15] R. Palmer, R. Fernow, and J. Lederman, Muon collider final cooling in 30-50 T solenoids, eConf. Proc. C110328, 2061 (2011).

[16] D. Neuffer, Introduction to muon cooling, Nucl. Instrum. Methods Phys. Res., Sect. A 532, 26 (2004).

[17] H. Bethe, Moliere's theory of multiple scattering, Phys. Rev. 89, 1256 (1953).

[18] R. B. Palmer, R. Weggel, and R. Fernow, Final cooling in 30-50 T solenoids, BNL, 2010 (unpublished).

[19] S. A. Kahn, in Proceedings of the 22nd Particle Accelerator Conference, PAC-2007, Albuquerque, NM (IEEE, New York, 2007).

[20] S. high field magnet designs and performances was reported in http://www.magnet.fsu.edu/.

[21] E. Bielert, J. Bremer, N. Doshita, R. Geyer, R. Hashimoto, N. Horikawa, S. Ishimoto, T. Iwata, K. Kondo, G. Mallot, H. Matsuda, T. Matsuda, Y. Miyachi, G. Nukazuka, O. Pirotte, H. Suzuki, S. Suzuki, and B. Vullierme, A $2.5 \mathrm{~m}$ long liquid hydrogen target for COMPASS, Nucl. Instrum. Methods Phys. Res., Sect. A 746, 20 (2014).

[22] H. K. Sayed, High intensity muon beam source for neutrino beam experiments, Nucl. Instrum. Methods Phys. Res., Sect. A 794, 193 (2015).

[23] T. J. Roberts and D. M. kaplan, in Proceedings of Particle Accelerator Conference, 2007 (IEEE, New York, 2007), pp. 3468-3470.

[24] S. Agostinelli, J. Allison, K. Amako, J. Apostolakis, H. Araujo, P. Arce, M. Asai, D. Axen, S. Banerjee, G. Barrand, F. Behner, L. Bellagamba, and S. Chauvie, Geant4a simulation toolkit, Nucl. Instrum. Methods Phys. Res., Sect. A 506, 250 (2003).

[25] J. Qiang, C. Mitchell, S. Paret, R. Ryne, and Y. Chao, A parallel multi-objective differential evolution algorithm for photoinjector beam dynamics optimization, in Proceedings, 4th International Particle Accelerator Conference (IPAC 2013) (2013), http://JACoW.org/IPAC2013/papers/ mopwo062.pdf. 